\title{
Perceived Gastrointestinal Symptoms and Association With Meals in a French Cohort of Patients With Irritable Bowel Syndrome
}

\author{
Chloé Melchior, ${ }^{1,2,3 *}$ Simon Fremaux ${ }_{4}^{4}$ Pauline Jouët, ${ }^{4}$ Gilles Macaigne, ${ }^{5}$ Jean-Jacques Raynaud, ${ }^{4}$ Suzelle Facon, ${ }^{6}$ Franck Iglicki, ${ }^{7}$ \\ Yannick Taes, ${ }^{8}$ and Jean-Marc Sabate ${ }^{4}$ \\ ${ }^{1}$ INSERM UMR 1073, Institute for Research and Innovation in Biomedicine, Normandy University, Rouen, France; ${ }^{2}$ Department of \\ Gastroenterology and INSERM CIC-CRB 1404, Rouen University Hospital, Rouen, France; ${ }^{3}$ Department of Molecular and Clinical Medicine, \\ Institute of Medicine, Sahlgrenska Academy, University of Gothenburg; Gothenburg, Sweden; ${ }^{4}$ Service de Gastroentérologie, Hôpital Avicenne \\ AP-HP, INSERM U-987, Bobigny, France; ${ }^{5}$ Department of Hepatogastroenterology, Marne-la-Vallee Hospital, Jossigny, France; ${ }^{6}$ The French \\ Association of IBS Patients (APSSII), Paris France; ${ }^{7}$ Gastroenterology Unit, AP-HP, Louis Mourier Hospital and Denis Diderot University Paris 7 , \\ Paris, France; and ${ }^{8}$ Alantaya creator, Paris France
}

\section{Background/Aims}

The aim of our study is to evaluate the association between meals and perceived gastrointestinal symptoms in real life in a French cohort of irritable bowel syndrome (IBS) patients.

\section{Methods}

This prospective cross-sectional observational study included patients from the French association (association des patients souffrant du syndrome de l'intestin irritable [APSSIII) of IBS. Data were collected on demographics, IBS subtype, dietary food, and meal-induced gastrointestinal symptoms from patient filled self-questionnaires or questionnaires.

\section{Results}

Eighty-four patients with IBS were included; $82.3 \%$ female with a mean age of $46.9 \pm 15.7$ years. Each transit pattern subtype represented one-third of the population. Forty-five percent of patients had severe IBS according to IBS-Severity Scoring System; mean IBS Quality of Life score was $53.9 \pm$ 18.3. Patients believed that food could trigger or exacerbate gastrointestinal symptoms in $73.3 \%$ and $93.4 \%$, respectively. Eighty-nine percent had already tried diets, mostly lactose free diet and low fermentable, oligosaccharides, disaccharides, monosaccharides, and polyols diet in $61.3 \%$ and $53.6 \%$ of cases. Thirty-nine percent of meals induced gastrointestinal symptoms. Meal-induced gastrointestinal symptoms were associated with severity and subtype but not with quality of life.

\section{Conclusions}

This study has confirmed the important link between gastrointestinal symptoms and food. Gastrointestinal symptoms induced by meals are frequent and associated with severity and IBS-diarrhea subtype. Our study also underlines patients' interest in food and diet. More knowledge is needed on food that triggers IBS symptoms but also on diet conditions in order to improve this condition.

(J Neurogastroenterol Motil 2021;27:574-580)

Key Words

Food; Irritable bowel syndrome; Meals

Received: September 8, 2020 Revised: November 13, 2020 Accepted: December 2, 2020

() This is an Open Access article distributed under the terms of the Creative Commons Attribution Non-Commercial License (http://creativecommons. org/licenses/by-nc/4.0) which permits unrestricted non-commercial use, distribution, and reproduction in any medium, provided the original work is properly cited.

*Correspondence: Chloé Melchior, MD, PhD

Department of Gastroenterology, Rouen University Hospital, 1 rue de Germont, 76031 Rouen Cedex, France Tel: +33-232-886-707, Fax: +33-232-888-425, E-mail: chloe.melchior@chu-rouen.fr 
life in a French cohort of IBS patients.

\section{Introduction}

Irritable Bowel Syndrome (IBS) is one of the most frequent functional gastrointestinal (GI) disorders. IBS is defined according to the Rome IV criteria as chronic or recurrent abdominal pain associated with abnormal bowel habits. ${ }^{1}$ IBS is divided into 3 predominant subtypes according to the predominant transit: IBS with predominant constipation (IBS-C), IBS with predominant diarrhea (IBS-D), and mixed IBS (IBS-M). The prevalence of IBS is around $5.0 \%$ to $10.0 \%$ according to the classification used. In the French Nutrinet-Santé cohort ${ }^{2}$ the prevalence was $5.6 \%$ using the most recent Rome criteria (Rome IV) and around $10.0 \%$ in an older study using the previous Rome III criteria. The impact of IBS on the quality of life (QOL), in particular in patients with the most severe disease, representing one-quarter of all IBS patients, is significant in at least 5 domains of daily life. ${ }^{3}$

The pathophysiology of IBS is multifactorial and incompletely understood. ${ }^{4}$ Altered gut-brain interactions, visceral hypersensitivity, psychosocial distress, GI motor disturbances, low-grade inflammation, intestinal immune activation, increased intestinal permeability, genetic and altered microbiome can all contribute to symptom generation in IBS. This heterogeneity of IBS presentation makes it difficult to treat and to improve the symptoms of individual IBS patients.

There is also a growing interest in the role of food in symptom generation. ${ }^{5}$ Meals and different food items (foods containing carbohydrates and fat) have been identified by patients as triggers of GI symptoms. When patients believe their symptoms to be related to food, they change their diet by avoiding the foods that they perceive as symptomatic. ${ }^{6}$ Food-related GI symptoms have been associated with more severe disease and reduced quality of life.

The current interest in the dietary management of IBS symptoms has largely focused on the negative effect of fermentable, oligosaccharides, disaccharides, monosaccharides, and polyols (FODMAP). ${ }^{8}$ These unabsorbed carbohydrates can generate IBS symptoms, suggesting the feasibility of a low FODMAP diet in IBS management. A low FODMAP diet could improve up to half of IBS patients.'

Most of these studies were performed in North Europe or Australia. Their results are difficult to generalize in France where food habits are different. Indeed, food shopping habits ${ }^{10}$ and food cooking practices are different between northern and southern European countries. ${ }^{11,12}$ Therefore, the aim of our study is to evaluate the association between meals and perceived GI symptoms in real

\section{Materials and Methods}

\section{Study Protocol}

This prospective cross-sectional observational study was conducted in France. All patients from the French Association of IBS patients (APSSII) who had agreed to participate in studies in accordance with the General Data Protection Regulation were contacted by e-mail and asked to participate in this study by answering anonymous online surveys between October and November 2017. The inclusion criteria were a clinically confirmed diagnosis of IBS according to the Rome IV criteria. Patients with incomplete surveys were not included. There were no exclusion criteria.

\section{Ethics Statement}

The agreement of the French Data Protection Authority (Commission Nationale de l'Informatique et des Libertés) was obtained for this online study (No. C3F2291202I). Privacy of patient information was ensured by password-protected access to the data collection forms, recording no information that could reveal the identity of individual patients in the online response database, and storing the online database on a secured internet server. No financial compensation was given for study participation. A patient's response to the surveys was considered as written consent. Patient responders were presented with information of the study.

\section{Patients}

Demographic and socio-demographic data were recorded: sex, age, body mass index (BMI), professional status, civil status, and monthly income. Smoking and alcohol consumption were also noted. The presence of a regular physical activity (> 30 minutes/ week) was recorded. Surgical history and co-morbidities such as allergy, fibromyalgia, and dyspepsia were also recorded.

Patients were asked the date of their IBS diagnosis to calculate IBS duration. IBS was defined according to Rome IV criteria ${ }^{1}$ and assessed with standardized questionnaires: IBS-Severity Scoring System (IBS-SSS) for severity, ${ }^{13}$ IBS-QOL for QOL,${ }^{14}$ Hospital Anxiety and Depression Scale (HADS) for anxiety and depression, ${ }^{15}$ and Bristol stool scale for IBS subtype. ${ }^{16}$ Patients were asked whether they were followed by a general practitioner or by a gastroenterologist, and if they had consulted in the last 12 months. Patients were asked about stress and food as triggering factors.

Another part of the survey focused on the perceived link be- 
tween food/diet and IBS symptoms. Patients were first asked if they believed that diet could improve IBS symptoms (in general and for their own disease). They were also asked if they believed that food could trigger or exacerbate their GI symptoms. Other questions about diet difficulties, diet preferences and previous diets were also asked.

\section{Dietary Food and Gastrointestinal Symptoms}

Patients reported 4 days of meals: 3 consecutive days during the week and at least 1 day during the weekend and their related GI symptoms. This part of the study was time consuming and participation was voluntary.

Patients reported the type of meal: breakfast, lunch, dinner, collation, or snack; and the day of the meal; weekday or weekend day. They had to report the day and time of each meal.

Patients reported GI symptoms for each meal. For this part, patients had to answer the question "Did you have gastrointestinal symptoms following this meal?" and if the answer was yes, then they had to report the presence of symptoms as abdominal pain, bloating, flatulence, and stools, as well as the time of the symptoms. Only 1 symptom could be reported for each type of symptom for each meal. For example, the patient was not able to report abdominal pain 3 times following the same meal but she/he could report abdominal pain once and bloating once for the same meal.

\section{Statistical Methods}

Data are expressed as percentage $(95 \% \mathrm{CI})$ and mean $\pm \mathrm{SD}$. Differences between subgroups were analyzed by Chi-squared test and correlation by Spearman test. Associations were considered statistically significant when $P<0.05$. The analysis was done using SPSS Statistics version 25.0 (IBM Corp, Armonk, NY, USA).

Table 1. Irritable Bowel Syndrome Socio Demographic Data

\begin{tabular}{lc}
\hline Socio-demographic data & Percentage \\
\hline In a relationship & $69.0 \%$ \\
With one or more children & $22.6 \%$ \\
Active employment & $63.1 \%$ \\
Monthly income & \\
$<1000$ euros & $5.7 \%$ \\
1000-2000 euros & $50.9 \%$ \\
2000-3000 euros & $24.5 \%$ \\
$>3000$ euros & $18.9 \%$ \\
Educational level & \\
Middle school & $3.6 \%$ \\
High school & $22.6 \%$ \\
Higher education & $73.8 \%$ \\
\hline
\end{tabular}

\section{Results}

\section{Patients}

A total of 84 IBS patients were included. Patients were mostly women $(\mathrm{n}=69,82.3 \%)$ with a mean age of $46.9 \pm 15.7$ years and a BMI of $21.7 \pm 3.7 \mathrm{~kg} / \mathrm{m}^{2}$.

Most of the patients were working $(63.1 \%)$, had a high level of education $(73.8 \%)$, and were in a relationship (69.0\%) (Table 1$)$.

Patients were active smokers in $15.5 \%$ and $14.3 \%$ had a minimal-moderate consumption of alcohol. In our population, 76.2\% had a regular physical activity. Patients had allergy history in $52.4 \%$ with a drug allergy in $23.8 \%$, allergic rhinitis in $36.9 \%$, eczema in $20.2 \%$, and asthma in $10.7 \%$. Fibromyalgia was associated in $11.9 \%$ and dyspepsia in $53.6 \%$.

Socio-demographic data are presented in Table 1.

\section{Irritable Bowel Syndrome}

The mean disease duration was $8.0 \pm 10.6$ years. Colonoscopy was performed in $81.0 \%$ of patients. In the previous 12 months, $83.3 \%$ of patients had consulted for their disease. IBS subtypes were diarrhea, constipation, and mixed-type in around one-third respectively and $44.6 \%$ of the patients had severe disease. Anxiety was reported in $38.1 \%$ of patients, with stress as a triggering factor in $69.0 \%$. QOL was altered in each of the 8 dimensions and mean IBS-QOL score was $53.9 \pm 18.3$. Other IBS characteristics are presented in Table 2.

Table 2. Irritable Bowel Syndrome Characteristics

\begin{tabular}{lc}
\hline \multicolumn{1}{c}{ IBS characteristics } & Percentage \\
\hline IBS subtype & \\
Diarrhea & $33.8 \%$ \\
Constipation & $32.5 \%$ \\
Mixed & $33.8 \%$ \\
IBS-SSS & \\
Severe $(>300)$ & $44.6 \%$ \\
Moderate $(175-300)$ & $33.8 \%$ \\
Mild (75-175) & $18.9 \%$ \\
Remission $(<75)$ & $2.7 \%$ \\
HAD depression $>10$ & $15.0 \%$ \\
HAD anxiety $>10$ & $38.1 \%$ \\
Stress triggering symptoms & $69.0 \%$ \\
Food triggering symptoms & $79.8 \%$ \\
\hline
\end{tabular}

IBS, irritable bowel syndrome; IBS-SSS, irritable bowel syndrome-severity scoring system; HADS, Hospital Anxiety and Depression Scale. 


\section{Food and Diet}

Patients believed that food could trigger or exacerbate GI symptoms respectively in $73.3 \%$ and $93.4 \%$, and that diet could improve IBS symptoms in general and for their own symptoms in $83.3 \%$ and in $65.5 \%$ of cases. Patients considered diet as an easy treatment to follow in daily life in $78.6 \%$ of cases. Ninety percent of the patients had already followed a diet, the diets used are presented

Table 3. Dietary Characteristics and Patients' Preferences for Diet Supervision

\begin{tabular}{lc}
\hline \multicolumn{1}{c}{ Dietary characteristics } & Percentage \\
\hline Patients having followed a diet & $89.3 \%$ \\
Type of diet & \\
Lactose free diet & $61.3 \%$ \\
Low FODMAP diet & $53.6 \%$ \\
Gluten free diet & $44.0 \%$ \\
High fiber diet & $21.3 \%$ \\
Elimination diet for allergy & $10.7 \%$ \\
Vegetarian diet & $9.3 \%$ \\
Vegan diet & $4.0 \%$ \\
Patients preferences for diet supervision & \\
Dietician & $70.2 \%$ \\
Physician & $56.0 \%$ \\
Website & $53.6 \%$ \\
Mobile application & $52.4 \%$ \\
Book & $50.0 \%$
\end{tabular}

FODMAP, fermentable, oligosaccharides, disaccharides, monosaccharides, and polyols.

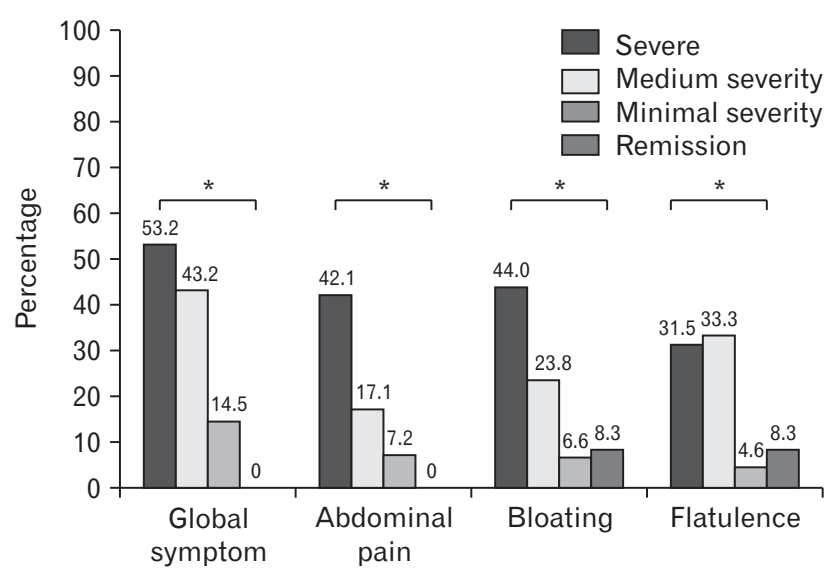

Fiǵure 2. Prevalence of perceived gastrointestinal symptoms induced by meals according to irritable bowel syndrome (IBS) severity. Severe: IBS-severity scoring system (IBS-SSS) > 300, moderate: IBS-SSS between 300 and 175, mild: IBS-SSS between 175 and 75, remission: IBS-SSS $<75 .{ }^{*} P<0.001$. in Table 3. The diets most used were lactose free diet (61.3\%) and low FODMAP diet (53.6\%) (Table 3). Patients preferred to be supervised by a dietician as shown in Table 3 .

\section{Dietary Food and Gastrointestinal Symptoms}

GI symptoms following meals were recorded and analyzed for 59/84 patients corresponding to 590 meals during 194 days (69.6\% during weekdays and $30.4 \%$ during weekend days). These 59 patients had similar characteristics to the 84 patients of the cohort. $38.6 \%$ of meals induced GI symptoms. The number of days with (at least 1 symptom) or without GI symptoms was not different between weekdays and weekend days (71.9\% vs $69.5 \%)$.

Regarding the type of meal, dinner, lunch, and breakfast induced more frequent symptoms than collation and snacks $(P<$

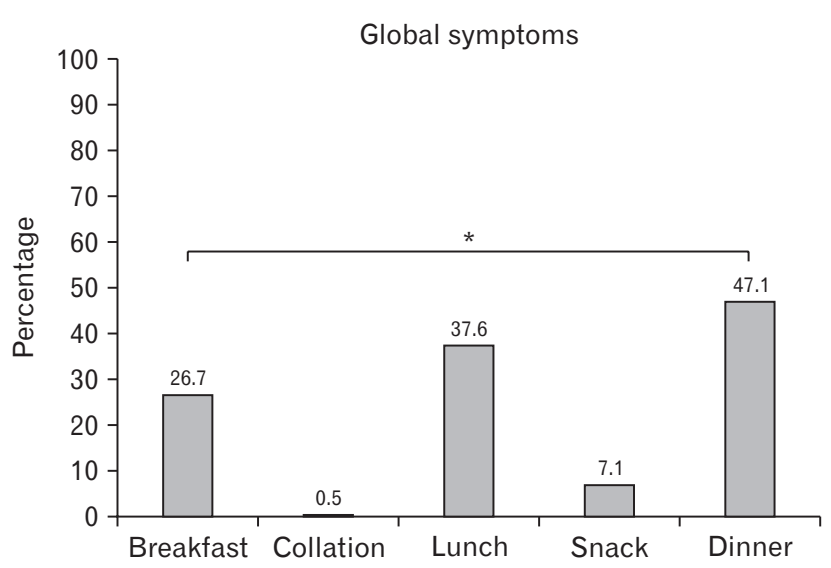

Figure 1. Prevalence of self-reported global gastrointestinal symptoms induced by meals according to type of meal. ${ }^{*} P<0.001$.

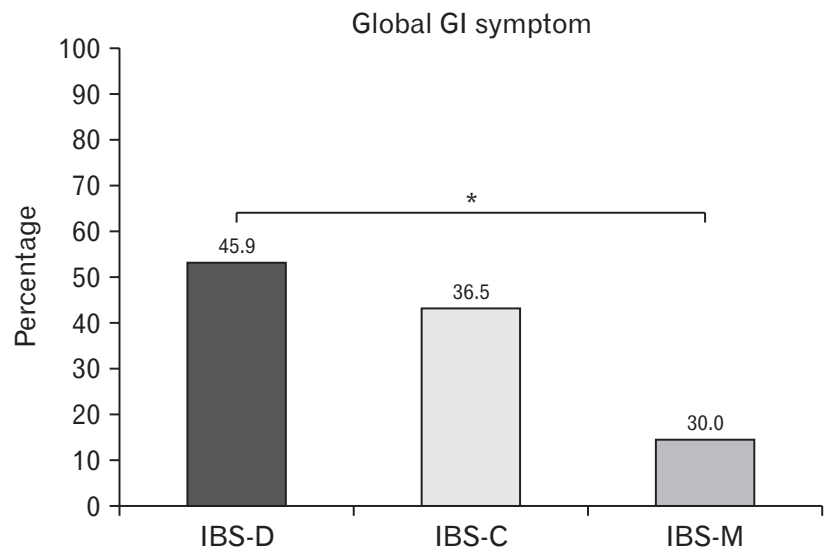

Figure 3. Prevalence of perceived global gastrointestinal (GI) symptoms induced by meals according to irritable bowel syndrome (IBS) subtype. IBS-D, IBS with predominant diarrhea; IBS-C, IBS with predominant constipation; IBS-M, mixed IBS. ${ }^{*} P=0.019$. 
0.001; Fig. 1).

Global GI symptoms induced by meals were more frequent according to IBS severity (Fig. 2). Individual GI symptoms as "abdominal pain," "bloating," and "flatulence" were more frequent according to IBS severity ( $P<0.001$; Fig. 2). There was no difference for the symptom "meal-induced stools" according to the degree of severity (severe: $36.6 \%$, moderate: $41.0 \%$, mild: $35.5 \%$, and remission: $33.3 \% ; P=0.713$ ). There was no correlation between the number of GI symptoms induced by meals and QOL ( $P=$ $0.900)$.

GI symptoms induced by meals were more frequent in IBSD subtype (IBS-D: $45.9 \%$, IBS-C: $36.5 \%$, and IBS-M: 30.0\%, P= 0.019 ) (Fig. 3), and no difference was found for "meal-induced stools" according to subtype $(38.6 \%, 29.5 \%$, and $40.8 \%$ for IBS-D, IBS-C, and IBS-M; $P=0.057)$. There was no difference in GI symptoms induced by meals according to associated comorbidities, as functional dyspepsia $(P=0.120)$.

\section{Discussion}

Several international studies have assessed the role of food on GI symptoms and food habits. IBS patients are known to adapt their diet according to their symptoms. ${ }^{17}$ All these studies report the importance of food on GI symptoms, and the frequent use of elimination diets by IBS patients. In the French Nutrinet-Santé cohort, 2423 IBS patients were classified in 3 major dietary patterns: healthy, traditional, and western $\operatorname{diet}^{18}$ This study underlined the role of fatty and sugary products in IBS, and found a strong association between western diet and IBS in this cohort. ${ }^{18}$ Unfortunately, the use of a specific elimination diet was not analyzed in this study. ${ }^{18}$ The role of sugar such as fructose and fat in IBS symptoms has already been highlighted in the past. ${ }^{5,19}$

Therefore, an observational study in real life in France is useful for a better understanding of the relation between meals and GI symptoms. Indeed, the results of international studies are difficult to extrapolate to French dietary habits. Our study is the first to assess in France the direct impact of meals on GI symptoms in an IBS population. In our population, $79.8 \%$ of patients declared that food triggered their symptoms with $38.6 \%$ of meals inducing GI symptoms with no difference between week and weekend days. This rate is higher than that obtained in Sweden, in a cohort of 330 patients with $63.0 \%$ of patients with food-related GI symptoms. ${ }^{5}$ It could be due to a recruiting bias. Indeed, the IBS patients of our French association des patients souffrant du syndrome de l'intestin irritable (APSSII) were asked to participate in this study by e-mail.
This voluntary participation could have led to a higher representation of patients with food-related symptoms and severe disease. The other explanation is the growing interest of patients and physicians in food and diet in IBS over the last decade. For example, in France, in 2013 , only $45.9 \%$ of the IBS patients of our French association (association des patients souffrant du syndrome de l'intestin irritable [APSSII]) were following a diet at the time of the study compared to $78.3 \%$ in $2020 .^{20}$ IBS patients wished to have more information on diet because they believe that elimination diets may improve their symptoms. ${ }^{21,22}$ This need for more information on elimination diets seems to be less pronounced in countries in which diets are used in first intention. ${ }^{23}$ Another point is that light meals (snack and collation) were less associated with GI symptoms than other meals (breakfast, lunch, and dinner). In Sweden, people eat lighter and healthier meals than in France, which could explain the difference between meal-induced symptoms between France and Sweden.

Most GI symptoms induced by meals were associated with severity but not with QOL in our study. Böhn et $\mathrm{al}^{7}$ found the same results for severity but found an association with impaired QOL. Probably our results lack power while 200 patients were analyzed in the Böhn study. ${ }^{7}$ It would have been interesting to use another tool, the more specific food related QOL. ${ }^{24}$ Indeed, food related QOL measures the impact of diet, eating behavior and food-related anxiety and is probably more relevant to measure the impact of foodrelated symptoms and diet on IBS QOL. ${ }^{25}$ Another interesting point is that global GI symptoms induced by meals, as abdominal pain, bloating, and flatulence, were associated with IBS subtype. Symptoms induced by meals were more frequent in IBS-D than in IBS-C and IBS-M. This result was not found for the symptom "meal-induced stools" which is surprising, since IBS-D was more involved. However, this could be due to the fact that symptoms were self-reported using the Bristol stool scale, and also to the role of visceral hypersensitivity. Simrén et $\mathrm{al}^{5}$ also found a link between symptoms induced by food and IBS subtype for some food items. There are unfortunately very few data in the literature according to this result.

Our recruiting method resulted in a population with more severe disease, interested in diet and with a high social level. Regarding sex ratio, BMI, age, and subtype these results are similar to those in the literature. ${ }^{5,7,18,26}$ IBS was severe in our population, with less than $3.0 \%$ of patients considered in remission. As expected, severity was associated with impaired QOL. ${ }^{27}$ Regarding associated comorbidities, functional dyspepsia was reported in $53.6 \%$ and fibromyalgia in $11.9 \% .^{22,28} \mathrm{~A}$ history of allergy was highly represented in our population $(52.4 \%)$ in comparison with $30.0 \%$ of atopy in 
the French population. A correlation is already known between atopy and IBS. ${ }^{29}$ Tobacco and alcohol were underrepresented in our population, respectively $15.5 \%$ and $14.3 \%$. The same results were found in the Nutrinet-Santé cohort ${ }^{18}$ and could be explained by the impact of tobacco and alcohol on the intestine.

Most of our patients had already followed a diet as expected with our recruiting bias. An interest in low FODMAP diet and the recent recommendations for its use in IBS patients ${ }^{30}$ probably led to an increase in the number of patients following this diet. Indeed, in 2013, only $9.0 \%$ of all patients following a diet were on a low FODMAP $\operatorname{diet}^{20}$ in comparison with $53.6 \%$ in this study. The lactose free diet and the gluten free diet were also frequently used, which is interesting as they both excluded the FODMAP subtype. This was not surprising for the lactose free diet since lactose intolerance is very common in France, ${ }^{31}$ For the gluten free diet, it was probably a trend with the recent development of gluten free products. Unfortunately, we do not have enough information on the conditions of these diets or of their perceived efficacy. It is likely that social media have helped the diffusion of these diets among patients and patients' associations.

In our population, $23.2 \%$ of patients had a low BMI. We did not record patients' history of eating disorders. This result could be related to associated eating disorders. Before prescribing a diet in IBS, physicians should always search for an underlying eating disorder, which is present in one in four of our patients and is associated with severity and impaired QOL. ${ }^{32}$ In particular, eating disorders are associated with a better adherence to low FODMAP diet in IBS. ${ }^{33}$

Even if there is a better knowledge today of food that triggers GI symptoms in IBS, food still plays a key role in this pathology and warrants further investigation.

In conclusion, this study conducted among patients from the French association of IBS patients has confirmed the important link between GI symptoms and food. GI symptoms induced by meals are frequent and associated with severity and IBS-D subtype. Our study also underlines patients' interest in food and diet. Lactose free diet and low FODMAP diet are the most frequently used. More knowledge is needed on food that triggers IBS symptoms but also on diet conditions to improve IBS treatment.

Acknowledgements: The authors are grateful to Nikki Sabourin-Gibbs, Rouen University Hospital, for her help in editing the manuscript. This study was realized with the French association of IBS Patients (APSSII for "Association Française des Patients Souffrant du Syndrome de l'Intestin Irritable," website www.apssii.org)
Financial support: The study was supported by Alantaya.

Conflicts of interest: None.

Author contributions: Conceptualization: Yannick Taes, Pauline Jouët, Jean-Marc Sabate, Simon Fremaux, Gilles Macaigne, Jean-Jacques Raynaud, and Franck Iglicki; data curation: Simon Fremau, Jean-Marc Sabat, and Yannick Taes; formal analysis: Suzelle Facon, Pauline Jouët, Chloé Melchior, and Jean-Marc Sabate; supervision: Jean-Marc Sabate; and writing: Chloé Melchior, Pauline Jouët, and Jean-Marc Sabate. All authors validated, reviewed, and edited the manuscript.

\section{References}

1. Mearin F, Lacy BE, Chang L, et al. Bowel disorders. Gastroenterology 2016;150:1393-1407, e5.

2. Le Pluart D, Sabaté JM, Bouchoucha M, Hercberg S, Benamouzig R, Julia C. Functional gastrointestinal disorders in 35,447 adults and their association with body mass index. Aliment Pharmacol Ther 2015;41:758767.

3. Ballou S, Keefer L. The impact of irritable bowel syndrome on daily functioning: characterizing and understanding daily consequences of IBS. Neurogastroenterol Motil 2017;29:e12982.

4. Ford AC, Lacy BE, Talley NJ. Irritable bowel syndrome. N Engl J Med 2017;376:2566-2578.

5. Simrén M, Månsson A, Langkilde AM, et al. Food-related gastrointestinal symptoms in the irritable bowel syndrome. Digestion 2001;63:108115 .

6. Hayes P, Corish C, O'Mahony E, Quigley EM. A dietary survey of patients with irritable bowel syndrome. J Hum Nutr Diet 2014;27(suppl 2):36-47.

7. Böhn L, Störsrud S, Törnblom H, Bengtsson U, Simrén M. Selfreported food-related gastrointestinal symptoms in IBS are common and associated with more severe symptoms and reduced quality of life. Am J Gastroenterol 2013;108:634-641.

8. Gibson PR, Shepherd SJ. Evidence-based dietary management of functional gastrointestinal symptoms: the FODMAP approach. J Gastroenterol Hepatol 2010;25:252-258.

9. Böhn L, Störsrud S, Liljebo T, et al. Diet low in FODMAPs reduces symptoms of irritable bowel syndrome as well as traditional dietary advice: a randomized controlled trial. Gastroenterology 2015;149:1399-1407, e2.

10. Pettinger C, Holdsworth M, Gerber M. 'All under one roof?' differences in food availability and shopping patterns in Southern France and Central England. Eur J Public Health 2008;18:109-114.

11. Pettinger C, Holdsworth M, Gerber M. Psycho-social influences on food choice in Southern France and Central England. Appetite 2004;42:307-316.

12. Pettinger C, Holdsworth M, Gerber M. Meal patterns and cooking practices in Southern France and Central England. Public Health Nutr 
2006;9:1020-1026.

13. Francis CY, Morris J, Whorwell PJ. The irritable bowel severity scoring system: a simple method of monitoring irritable bowel syndrome and its progress. Aliment Pharmacol Ther 1997;11:395-402.

14. Patrick DL, Drossman DA, Frederick IO, DiCesare J, Puder KL. Quality of life in persons with irritable bowel syndrome: development and validation of a new measure. Dig Dis Sci 1998;43:400-411.

15. Cho HS, Park JM, Lim CH, et al. Anxiety, depression and quality of life in patients with irritable bowel syndrome. Gut Liver 2011;5:29-36.

16. Heaton KW, Radvan J, Cripps H, Mountford RA, Braddon FE, Hughes AO. Defecation frequency and timing, and stool form in the general population: a prospective study. Gut 1992;33:818-824.

17. Torres MJ, Sabate JM, Bouchoucha M, Buscail C, Hercberg S, Julia C. Food consumption and dietary intakes in 36,448 adults and their association with irritable bowel syndrome: Nutrinet-Santé study. Therap Adv Gastroenterol 2018;11:1756283X17746625.

18. Buscail C, Sabate JM, Bouchoucha M, et al. Western dietary pattern is associated with irritable bowel syndrome in the french nutrinet cohort. Nutrients 2017;9:986.

19. Melchior C, Douard V, Coëffier M, Gourcerol G. Fructose and irritable bowel syndrome. Nutr Res Rev 2020;33:235-243.

20. Sabaté JM, Ducrott P, Piche T, et al. Expectations of IBS patients concerning disease and healthcare providers: results of a prospective survey among members of a French patients' association. Clin Res Hepatol Gastroenterol 2020;44:961-967.

21. Halpert A, Dalton CB, Palsson O, et al. What patients know about irritable bowel syndrome (IBS) and what they would like to know. National survey on patient educational needs in IBS and development and validation of the patient educational needs questionnaire (PEQ). Am J Gastroenterol 2007;102:1972-1982.

22. Olafsdottir LB, Gudjonsson H, Jonsdottir HH, Jonsson JS, Bjornsson E, Thjodleifsson B. Irritable bowel syndrome: physicians' awareness and patients' experience. World J Gastroenterol 2012;18:3715-3720.

23. Bijkerk CJ, de Wit NJ, Stalman WA, Knottnerus JA, Hoes AW, Muris
JW. Irritable bowel syndrome in primary care: the patients' and doctors' views on symptoms, etiology and management. Can J Gastroenterol 2003;17:363-368.

24. Hughes LD, King L, Morgan M, et al. Food-related quality of life in inflammatory bowel disease: development and validation of a questionnaire. J Crohns Colitis 2016;10:194-201.

25. Guadagnoli L, Mutlu EA, Doerfler B, Ibrahim A, Brenner D, Taft TH. Food-related quality of life in patients with inflammatory bowel disease and irritable bowel syndrome. Qual Life Res 2019;28:2195-2205.

26. Lovell RM, Ford AC. Global prevalence of and risk factors for irritable bowel syndrome: a meta-analysis. Clin Gastroenterol Hepatol 2012;10:712-721, e4.

27. Drossman DA, Patrick DL, Whitehead WE, et al. Further validation of the IBS-QOL: a disease-specific quality-of-life questionnaire. Am J Gastroenterol 2000;95:999-1007.

28. de Bortoli N, Tolone S, Frazzoni M, et al. Gastroesophageal reflux disease, functional dyspepsia and irritable bowel syndrome: common overlapping gastrointestinal disorders. Ann Gastroenterol 2018;31:639-648.

29. Jones MP, Walker MM, Ford AC, Talley NJ. The overlap of atopy and functional gastrointestinal disorders among 23471 patients in primary care. Aliment Pharmacol Ther 2014;40:382-391.

30. McKenzie YA, Bowyer RK, Leach H, et al. British dietetic association systematic review and evidence-based practice guidelines for the dietary management of irritable bowel syndrome in adults (2016 update). J Hum Nutr Diet 2016;29:549-575.

31. Szilagyi A, Ishayek N. Lactose intolerance, dairy avoidance, and treatment options. Nutrients 2018;10:1994.

32. Melchior C, Desprez C, Riachi G, et al. Anxiety and depression profile is associated with eating disorders in patients with irritable bowel syndrome. Front Psychiatry 2020;10:928.

33. Mari A, Hosadurg D, Martin L, Zarate-Lopez N, Passananti V, Emmanuel A. Adherence with a low-FODMAP diet in irritable bowel syndrome: are eating disorders the missing link? Eur J Gastroenterol Hepatol 2019;31:178-182. 